\title{
NASA TECHNICAL
}

MEMORANDUM

\%

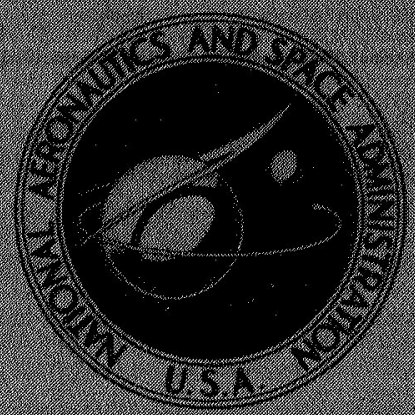

NASA TM X-1618

GPO PRICE

CFSTI PRICE(S) \$

Hard copy $(\mathrm{HC})$

3,4

N $68-31498$

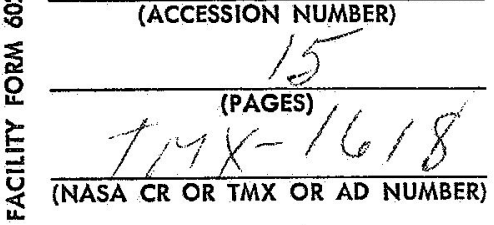

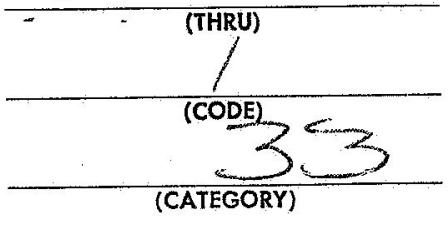

Microfiche (MF)

.65

\& 853 July 65

EXPERIMENTAL MEASUREMENTS

OF THERMAL RADIATION PROPERTIES

BY CYCIIC INCIDENT RADIATION

by Ermie W. Spisz and John R. Jack

Lewis Research Center

Cleveland, Obio

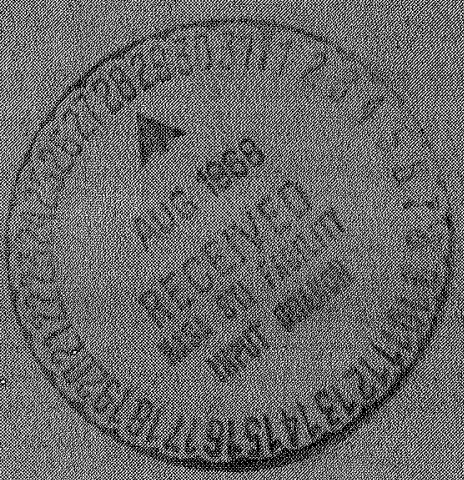

national aeronautics and SPaCe administration - washington, D. C. • AUGUSt 1968 
NASA TM X-1618

EXPERIMENTAL MEASUREMENTS OF THERMAL RADIATION PROPERTIES BY CYCLIC INCIDENT RADIATION

By Ernie W. Spisz and John R. Jack

Lewis Research Center Cleveland, Ohio

\section{NATIONAL AERONAUTICS AND SPACE ADMINISTRATION}

For sale by the Clearinghouse for Federal Scientific and Technical Information Springfield, Virginia 22151 - CFSTI price $\$ 3.00$ 
A new experimental technique is developed to determine simultaneously the total hemispherical emittance, solar absorptance, and absorptance-emittance ratio for metals at cryogenic temperatures. The method compares the temperature response of thin metal samples to a sinusoidal perturbation of an imposed radiant intensity. Emittance data are presented for aluminum, copper, and 304 stainless steel over the temperature range of 150 to $500 \mathrm{~K}$. From the data, the applicability of the cyclic radiant intensity method is evaluated, and the problems associated with extending the method to temperatures below $150 \mathrm{~K}$ are discussed. 


\title{
EXPERIMENTAL MEASUREMENTS OF THERMAL RADIATION PROPERTIES BY CYCLIC INCIDENT RADIATION
}

\author{
by Ernie W. Spisz and John R. Jack \\ Lewis Research Center
}

\section{SUMMARY}

A new experimental technique is presented for determining simultaneously the total hemispherical emittance, solar absorptance, and absorptance-emittance ratio $\alpha / \epsilon$ for metals at cryogenic temperature. The method compares the temperature response of a thin metal sample to a sinusoidal perturbation of the imposed radiant intensity. Emittance data are presented for aluminum, copper, and 304 stainless steel over the temperature range of 150 to $500 \mathrm{~K}$. The cyclic-radiant-intensity method was convenient for providing radiation property data of most metals for temperatures above $150 \mathrm{~K}$. The primary difficulty encountered in extending the technique to temperatures below $150 \mathrm{~K}$ is the precise measurement of the phase angle between the sample temperature and the sinusoidal intensity perturbation. The Lissajous figure method used herein is accurate for phase angles up to seventy degrees. However, at temperatures below $150 \mathrm{~K}$, phase angles greater than seventy degrees are encountered for most low-emittance metals, and more refined methods of phase angle measurement are required. Other problem areas that require consideration are the long time periods (greater than $3 \mathrm{hr}$ ) required to reach cyclic equilibrium and the precise radiant-intensity control required at the low-intensity levels.

\section{INTRODUCTION}

Many different methods are being used for determining experimentally the total hemispherical emittance, solar absorptance, and absorptance-emittance ratio $\alpha / \epsilon$ of various spacecraft materials. Each method has its advantages for particular situations, but none is sufficiently general or versatile to permit measurement of all three parameters simultaneously and accurately over a broad range of temperatures and material property values. One of the most difficult situations that is encountered in determining radiation 
properties occurs with pure metals (i.e., low emittance) at low temperatures. The experimental difficulties occur because of the small quantities of energy being exchanged and the correspondingly small changes in measurable parameters. As a result, various effects such as heat leaks and measurement errors, which are inherent in any experimental technique and data analysis, must be identified and evaluated. To minimize these possible errors requires that a specific technique be generally "tailored" to consider only a single radiation parameter over a limited temperature range.

A new method that compares the temperature response of a sample to a sinusoidal perturbation of an imposed radiant intensity is proposed in reference 1 . This cyclic method appears to overcome a number of the limitations of previous methods. For example, the total hemispherical emittance, solar absorptance, and $\alpha / \epsilon$ can be determined simultaneously over a wide temperature range with good accuracy. In addition, the method circumvents the experimental problems of sample heat leaks by permitting the heat leaks to exist and also to remain undefined, provided they are not temperature dependent over the range of the sample temperature perturbation. However, because the method is based on a cyclic perturbation, the control of the experiment (specifically, the radiant-intensity perturbation) and the analysis of the experimental data present unique problems. This report describes the experimental technique being developed to apply this method. Emittance data are presented over the temperature range of 150 to $500 \mathrm{~K}$, from which the applicability of method can be evaluated and the problems associated with extending the technique to temperatures below $150 \mathrm{~K}$ can be assessed.

\section{METHOD}

The cyclic-radiant-intensity method considers a thin metal sample of thickness $\tau$ carefully suspended in an ultrahigh vacuum, cold-wall environment. A steady radiant intensity $I_{0}$ is imposed upon the sample to establish an initial sample equilibrium temperature $T_{\mathbf{m}}$. Once equilibrium temperature conditions are achieved, the radiant intensity is perturbed sinusoidally with an amplitude $\mathrm{kI}_{\mathrm{o}}$ (where $\mathrm{k}<1.0$ ) and a frequency $\omega$.

The differential equation describing the temperature of an isothermal sample is taken as (ref. 1)

$$
\rho c_{p} \tau \dot{T}=\alpha I_{0}(1+k \sin \omega t)+q-2 \epsilon \sigma T^{4}
$$

Within the limits of the temperature perturbation, the material properties, specific heat $c_{p}$, absorptance $\alpha$, and emittance $\epsilon$ are considered independent of temperature and the $T^{4}$ term is linearized about $T_{m}$ as $T^{4}=4 T_{m}^{3} T-3 T_{m}^{4}$. If the heat leak term 
$q$ is constant over the temperature perturbation, the temperature response of the sample to the sinusoidally perturbed radiant intensity is given by

$$
T=T_{m}+A\left[\sin (\omega t-\varphi)+e^{-t / \theta^{\prime}} \sin \varphi\right]
$$

At cyclic equilibrium (i.e, when $\mathrm{e}^{-\mathrm{t} / \theta^{\prime}} \sin \varphi$ becomes negligible), the temperature response of the sample is sinusoidal around $\mathrm{T}_{\mathrm{m}}$ with amplitude $\mathrm{A}$ and phase angle $\varphi$.

The phase angle $\varphi$ is given by

$$
\varphi=\tan ^{-1} \omega \theta^{\prime}
$$

and the temperature amplitude by

$$
A=\left(\frac{\alpha I_{o} k}{\rho \tau c_{p}}\right)\left[\left(\frac{1}{\theta^{\prime}}\right)^{2}+\omega^{2}\right]^{-1 / 2}
$$

where $\theta^{\prime}$, the time constant of the material, is given by

$$
\theta^{\prime}=\frac{\rho \tau \mathrm{c}_{\mathrm{p}}}{8 \epsilon \sigma \mathrm{T}_{\mathrm{m}}^{3}}
$$

Equations (3) to (5) show that, for a given sample material and temperature level, the emittance and absorbtance are determined by the measured phase angle and temperature amplitude and by the imposed cyclic frequency, intensity level, and intensity perturbation amplitude. The mean sample temperature is established by the initial intensity level. The sample thickness, cyclic frequency, and intensity amplitude can be selected to provide the most favorable sample response from which accurate measurements of phase angle and temperature amplitude can be made. These characteristics along with the possibility of ignoring the heat leak term $q$ are the important advantages of the cyclic method.

\section{EXPERIMENTAL TECHNIQUE}

To determine radiation properties at low temperatures necessitates the use of a highvacuum, cold-wall facility to eliminate residual gas conduction and provide a low- 
temperature radiation sink. Figure 1 is a schematic drawing of the high-vacuum facility

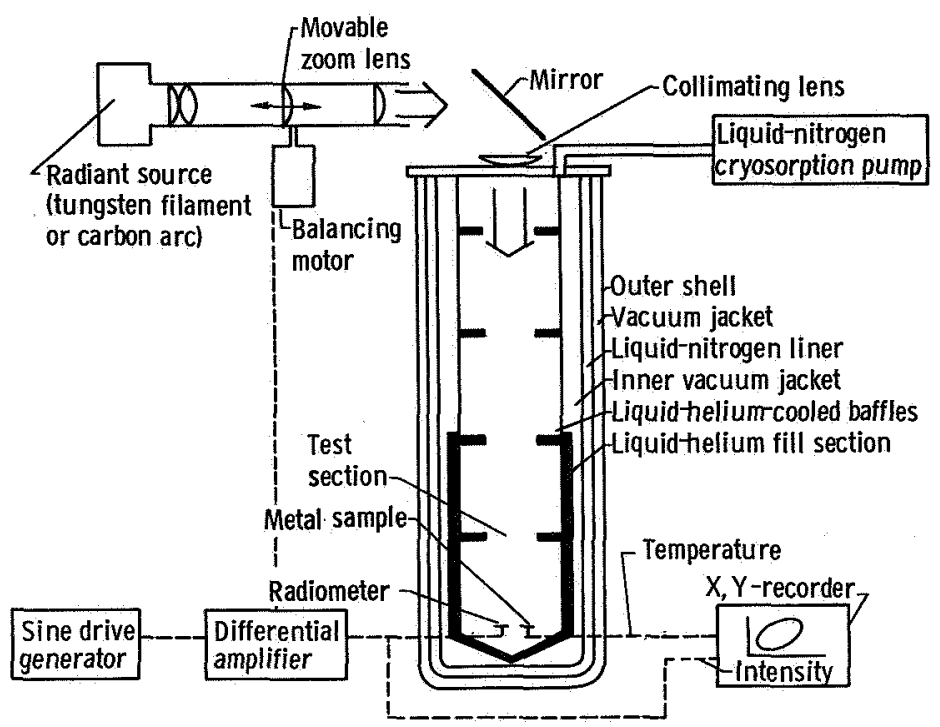

Figure 1. - Schematic drawing of experimental setup.

and the experimental arrangement used. The facility is the Solar Space Environment Simulator Facility described in reference 2. The original oil-diffusion and mechanical pumps have been replaced by liquid-nitrogen cryosorption pumps to eliminate oil backstreaming and possible contamination of the sample surfaces. The facility was initially pumped to a vacuum of $10^{-4}$ torr by the liquid-nitrogen cryosorption pumps. The annular walls of the test section and the baffles were then filled with liquid helium to cryopump the chamber to below $10^{-10}$ torr and to provide a radiation background of $4 \mathrm{~K}$. The four liquid-helium-cooled baffles within the test section eliminated stray radiation and permitted only the imposed collimated radiation to reach the test sample.

The test samples (generally $1 \mathrm{~cm}$ wide by $2 \mathrm{~cm}$ long) were carefully suspended in the test plane at the bottom of the test section by four 0.001 -inch- $(0.00254-\mathrm{cm}-)$ diameter wires. Two of the four wires are a matched Chromel-constantan pair that served as the sample thermocouple. The other two wires are either Chromel or constantan. The small-diameter alloy wire was used to minimize the effect of the wires on the sample response.

The radiation source was either a 12 -kilowatt carbon arc or a 1 -kilowatt quartziodine-tungsten filament lamp with associated collimating optics. The first zoom lens shown in figure 1 is capable of varying the radiant intensity by 10 percent about a mean value. Intensity measurements were made by either a solar cell or a thermopile radiometer located near the sample test plane. The sinusoidal radiant-intensity control was 
provided by coupling the radiometer output and the reference-sine-generator output into a differential amplifier that actuates a balancing motor to maintain the proper position of the movable zoom lens. The reference sine generator is variable and establishes the perturbation amplitude and cyclic frequency.

The data were recorded on a multichannel strip-chart recorder and an X, Yrecorder. The strip-chart recorder monitors the radiant intensity and sample thermocouples to ensure that the absolute intensity level and frequency are maintained. The $\mathrm{X}$, $\mathrm{Y}$-recorder is the primary data recording instrument. The reference sinusoidal signal (or the radiometer signal) is recorded on the $\mathrm{x}$-axis and the sample thermocouple on the y-axis. The resulting trace over one cycle provides an elliptical Lissajous figure (fig. 2)

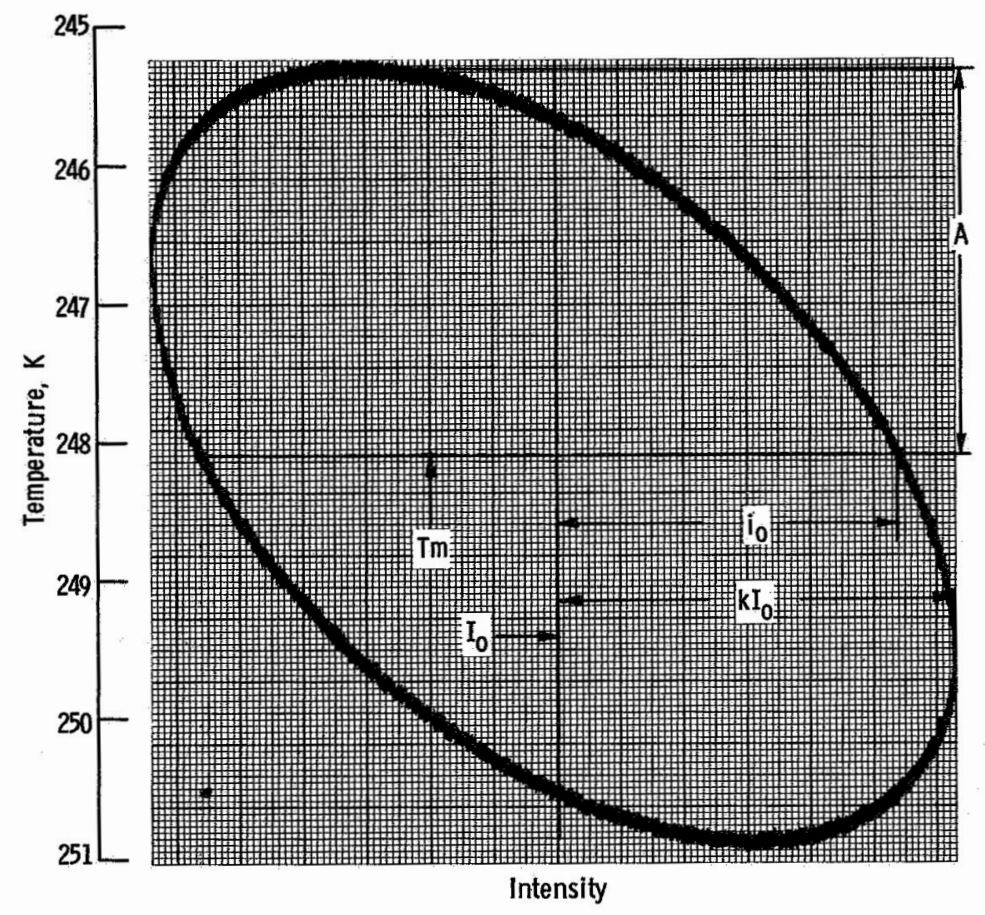

Figure 2. - X, Y-recorder figure for SS304 at 10 revolutions per hour.

from which the mean sample temperature, amplitude of the temperature oscillation, initial intensity level, and amplitude of the intensity perturbation can be obtained directly. In addition, the phase angle between the sample temperature and radiant intensity is (e.g., ref. 3)

$$
\varphi=\tan ^{-1}\left[\left(\frac{k I_{0}}{i_{0}}\right)^{2}-1\right]^{-1 / 2}
$$


where $i_{0}$ is the value of the intensity perturbation at the mean temperature. Figure 2 is a typical Lissajous figure obtained for a 0.001 -inch- $(0.00254-\mathrm{cm}-)$ thick 304 stainlesssteel sample with an incident radiation frequency of 0.01745 radian per second (10 $\mathrm{rph}$ ). The resulting figure is quite large with adequate resolution for an accurate measurement of $\mathrm{T}_{\mathrm{m}}, \mathrm{A}, \mathrm{I}_{\mathrm{o}}, \mathrm{i}_{\mathrm{o}}$, and $\mathrm{kI}_{0}$. The Lissajous figure also provides an additional monitoring function on the experiment. The figure must close and repeat when cyclic equilibrium is achieved. In figure 2 , which required 6 minutes to complete, even slight changes as small as one-tenth of a degree in the sample temperature can prevent closure and invalidate the data.

\section{RESULTS AND DISCUSSION}

The initial check made on the complete experimental system was to establish the linearity of the tangent of the phase angle with the cyclic radiation frequency (eq. (3)). Three different 0.001 -inch- $(0.00254-\mathrm{cm}-)$ thick metal samples with different material time constants were studied simultaneously. The materials selected were

(1) 304 Stainless steel with the as-received surface finish

(2) Commercially available aluminum foil with the as-received finish

(3) Rolled copper foil that was soaked in an ammonia-vinegar solution to provide a satin finish which would not oxidize readily

These materials were chosen because of their different time constants and correspondingly different response characteristics to provide a stringent test for the new method.

The 1-kilowatt quartz-iodine-tungsten-filament lamp was used as the radiation source because of its absolute stability over long time periods. The radiant-intensity level was established to provide mean sample temperatures of approximately $250 \mathrm{~K}$ and an intensity amplitude parameter $\mathrm{k}$ of approximately 0.1 . The cyclic frequency was varied from 0.00349 to 0.0262 radian per second (2 to $15 \mathrm{rph}$ ). These data are shown in figure 3. The slopes of the lines drawn through the data are the average values of the measured time constant $\theta^{\prime}$ (eq. (3)) for each material. The copper data for $\tan \varphi>5$ were discounted in determining the average value of $\theta^{*}$ because they depart from the linearity required by the analysis. This departure results from inaccuracies in determining the phase angle from the Lissajous figures. The relative error associated with evaluating $\tan \varphi$ by equation (6) is

$$
\frac{\delta(\tan \varphi)}{\tan \varphi}=\left(1+\tan ^{2} \varphi\right)\left[\frac{\delta\left(\mathrm{kI}_{0}\right)}{\mathrm{kI}_{0}}+\frac{\delta\left(\mathrm{i}_{0}\right)}{\mathrm{i}_{0}}\right]
$$




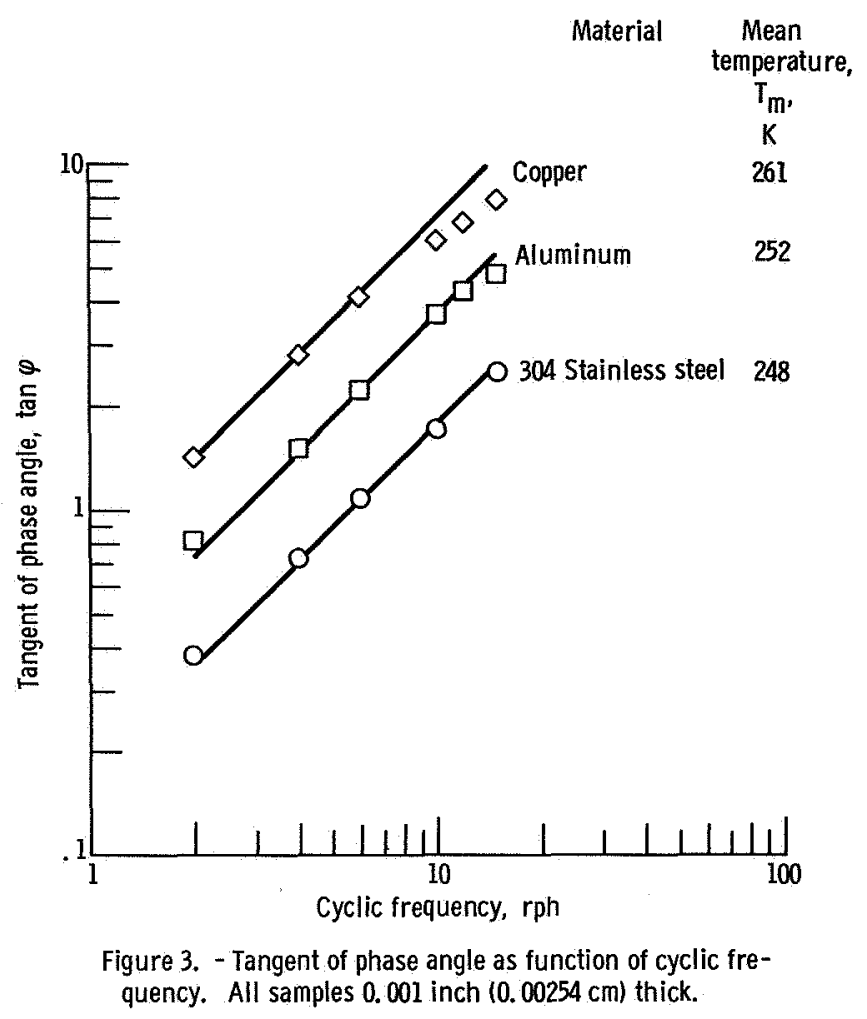

For $\tan \varphi=5$ and typical relative-intensity amplitude errors of $\delta\left(\mathrm{kI}_{\mathrm{o}}\right) / \mathrm{kI}_{\mathrm{o}}=0.01$ and $\delta\left(i_{0}\right) / i_{0}=0.02$, the relative error in $\tan \varphi$ is 78 percent and can easily account for the nonlinearity of the data.

The linearity of the remaining data indicates that the response of the sample temperature over the perturbation is described by the solution (eq. (2)) to the linearized differential equation. That is, the effect due to the radiation exchange $\mathrm{T}^{4}$ can be linearily approximated, and the temperature dependency of the material properties can be neglected.

The important assumption that requires the heat leak term $q$ to be constant over the perturbation cycle cannot be verified from these data because radiant-intensity measurements (which will be discussed later in this section) could not be made. However, from the linearity of the data and the symmetry of the Lissajous figures, this condition appears to be satisfied.

After it was established that the experimental technique would provide reproducible data independent of cyclic frequency, the radiant-intensity level was varied to obtain emittance data for the three samples over a range of temperatures. These data are presented in table I. The first four temperatures were obtained with the tungstenfilament lamp, and the final high-temperature data point was obtained with the carbon arc. 
TABLE I. - METAL SAM PLE DATA

\begin{tabular}{|c|c|c|c|c|c|c|}
\hline \multirow[t]{2}{*}{ Material } & \multicolumn{2}{|c|}{$\begin{array}{c}\text { Cyclic frequency, } \\
\omega\end{array}$} & \multirow{2}{*}{$\begin{array}{c}\text { Sample } \\
\text { temperature, } \\
\text { T }_{\mathbf{K}},\end{array}$} & \multirow{2}{*}{$\begin{array}{l}\text { Tangent } \\
\text { of phase } \\
\text { angle, } \\
\tan \varphi\end{array}$} & \multirow{2}{*}{$\begin{array}{c}\text { Material } \\
\text { time } \\
\text { constant, } \\
\theta^{\prime}, \\
\text { sec }\end{array}$} & \multirow[t]{2}{*}{$\begin{array}{c}\text { Emittance, } \\
\epsilon\end{array}$} \\
\hline & $\mathrm{rph}$ & $\mathrm{rad} / \mathrm{sec}$ & & & & \\
\hline \multirow{5}{*}{$\begin{array}{l}\text { 304 Stain- } \\
\text { less steel }\end{array}$} & 2.40 & 0.00419 & 175 & 1. 256 & 302 & 0.103 \\
\hline & 2.40 & .00419 & 201 & .743 & 177 & .126 \\
\hline & 2 to 15 & ----- & 248 & $-\cdots$ & $a_{102}$ & a. 129 \\
\hline & 4 & .00698 & 267 & .571 & 81.8 & .134 \\
\hline & $b_{12}$ & .0219 & 430 & .578 & 27.6 & .108 \\
\hline \multirow[t]{5}{*}{ Aluminum } & 2. 40 & 0.00419 & 170 & 2.45 & 585 & 0.0391 \\
\hline & 2.40 & .00419 & 200 & 1.73 & 413 & .0369 \\
\hline & 2 to 15 & ----- & 252 & ---- & $a_{210}$ & ${ }^{a} .0385$ \\
\hline & 4 & .00698 & 274 & 1. 197 & 171 & .0371 \\
\hline & $\mathrm{b}_{12}$ & .0219 & 458 & .996 & 47.5 & .0327 \\
\hline \multirow[t]{5}{*}{ Copper } & 2.40 & 0.00419 & 162 & 4. 38 & 1046 & 0.0379 \\
\hline & 2.40 & .00419 & 195 & 3.14 & 749 & .0319 \\
\hline & 2 to 15 & ----1 & 261 & $--\ldots$ & $a_{405}$ & a. 0263 \\
\hline & 4 & .00698 & 291 & 2.04 & 292 & .0266 \\
\hline & $\mathrm{b}_{12}$ & .0219 & 576 & .888 & 42.4 & .0270 \\
\hline
\end{tabular}

${ }^{a}$ Average values of data obtained with variable frequency from 0.00349 $0.0262 \mathrm{rad} / \mathrm{sec}(2$ to $15 \mathrm{rph})$, fig. 3 .

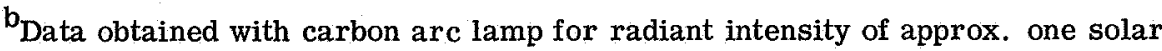
constant. All other data obtained with 1-kw-quartz-iodine-tungsten-filament lamp.

At each intensity level, the cyclic frequency was selected to provide a phase angle that would yield reasonably accurate emittance values. Because the data for the three samples were obtained simultaneously, the optimum cyclic frequency for each sample material could not be used. The phase angles for copper were somewhat high, whereas for 304 stainless steel they were slightly low. The phase angles required for accurate emittance values can be established by considering the relative error in emittance as determined from equations (3) and (5). If errors due to temperature and frequency measurement are neglected, the relative error in emittance is

$$
\frac{\delta(\epsilon)}{\epsilon}=\frac{\delta(\tan \varphi)}{\tan \varphi}
$$


That is, the emittance error is identical to the relative error in $\tan \varphi$ as given by equation (7).

The phase angle should be maintained between 0.7 and 1.22 radians to obtain reasonably accurate emittance values. As shown by equations (7) and (8), the emittance error for phase angles greater than 1.22 radians is governed by the tangent of the phase angle $\left(\tan ^{2} \varphi\right)$, whereas for phase angles less than 0.7 radian, the Lissajous figure becomes - slender and error is governed by the intensity amplitude errors.

The data obtained with the carbon arc were not as accurate as those obtained with the tungsten-filament lamp. Even though the phase angles are reasonable, the intensity amplitude errors due to arc instabilities, $\delta\left(\mathrm{kI}_{0}\right) / \mathrm{kI}_{0}$ and $\delta\left(\mathrm{i}_{\mathrm{o}}\right) / \mathrm{i}_{\mathrm{o}}$, are much larger than for the tungsten-filament lamp. The Lissajous figures for the carbon arc were "noisy" and, consequently, intensity amplitudes could not be measured accurately. The accuracy was further reduced because it was necessary to use part of the limited zoom-lens travel to compensate for drift in the mean intensity level of the carbon arc. The amplitude of the intensity perturbation is thereby reduced, and the ratio of noise to intensity amplitude is increased.

No noticeable trend of emittance with temperature was evidenced for any of the three materials for the data in table I. This result is consistent with other published data (refs. 4 and 5). The absolute value of emittance for these materials is also consistent with other published data.

The rapid increase of the time constant with desreasing temperature for the data in table $I$ is worth noting. Generally, the time required to achieve cyclic equilibrium is at least five times the material time constant. For the copper sample at the lowest temperature of $162 \mathrm{~K}$, the time constant is 1046 seconds. The cyclic frequency of 0.0042 radian per second $(2.4 \mathrm{rph}$ ) necessitated that absolute control of the radiant intensity be maintained for a period in excess of 2 hours to obtain a usable figure.

For temperatures above $150 \mathrm{~K}$, the time constants of most metals do not introduce difficult experimental problems. The cyclic-radiant-intensity method is easily applied, and good accuracy can be achieved without a very refined experimental technique. How ever, for temperatures below $150 \mathrm{~K}$, all low-emittance metals have time constants greater than 1000 seconds even for a sample thickness of 0.001 inch $(0.00254 \mathrm{~cm})$. Cyclic frequencies of 0.001745 radian per second $(1 \mathrm{rph})$ or less will be required to obtain the $\tan \varphi$ values necessary for good accuracy. Cyclic test times longer than 2 hours are required to reach cyclic equilibrium, and absolute control must be maintained over the complete experiment, particularly of the incident radiation. Any minor thermal disturbance during the cyclic period can result in sample temperature excursions with very slow recovery rates, which may require restart of the test.

A possible method of reducing the time constant of materials is to deposite a thin layer of the material on a substrate having an appropriate time constant. Materials such 
as molybdenum and lead could be used effectively without the introduction of large errors due to composite samples.

No attempt was made herein to obtain solar absorptance data for two reasons: first, evaluation of the solar absorptance requires the absolute value of the imposed radiantintensity level. An accurately calibrated radiometer was not available at the time, and only relative intensity measurements could be made. Second, the instabilities of the carbon arc lamp being used were beyond the capabilities of the automated control system, and they resulted in noisy Lissajous figures of small intensity amplitude. The consequences of a noisy trace and a small intensity amplitude on absorbtance data can be demonstrated by the relative error associated with the absorptance. The relative error is expressed as

$$
\frac{\delta(\alpha)}{\alpha}=2 \frac{\delta\left(\mathrm{kI}_{\mathrm{o}}\right)}{\mathrm{kI}_{\mathrm{o}}}+\frac{\delta\left(\mathrm{i}_{\mathrm{o}}\right)}{\mathrm{i}_{\mathrm{o}}}+\frac{\delta(\mathrm{A})}{\mathrm{A}}
$$

The relative absorptance error is governed entirely by the intensity - and temperatureamplitude measurements. For the carbon arc data, the amplitude measurements were only accurate to within 10 percent, which, according to equation (9), could have resulted in errors up to 40 percent for solar absorptance data. Both of these shortcomings in the present experimental apparatus are being remedied with improved equipment.

One interesting point with regard to absorptance measurements is that not only is the absorptance error independent of the phase angle, but the absorptance becomes independent of the material time constant for large time constant materials or high cyclic frequencies (eqs. (4) and (5)). Thus, if only absorptance data are required, the method is limited only by the difficulties imposed by the control of the radiation source. Since more stable arcs are now available, the technique is ideally suited for determining solar absorptance over a very large temperature range.

\section{SUMMARY EVALUATION OF CYCLIC METHOD}

The cyclic-radiant-intensity method is convenient and useful for determining radiation properties of most metals above temperatures of $150 \mathrm{~K}$. As indicated herein, accurate emittance data can be obtained over a temperature range from 150 to $500 \mathrm{~K}$ without much difficulty. Even though solar absorptance data are not presented because of difficulties with the measurement of intensity level and the control of the carbon arc source, the capability of the cyclic method to provide absorptance data easily and more accurately than emittance data seems assured. 
The primary difficulty encountered in extending the technique to temperatures below $150 \mathrm{~K}$ is the phase angle measurement. The Lissajous figure method used herein is quite applicable and accurate for most metals above temperatures of $150 \mathrm{~K}$ if the phase angle can be maintained between 0.70 and 1.22 radians by the proper selection of sample thickness or cyclic frequency. However, at temperatures below $150 \mathrm{~K}$, practical limitations on sample thickness and cyclic frequency can result in phase angles beyond the limits acceptable for the Lissajous figure. These limits, however, should not imply a limitation of the cyclic method. Accurate phase angle measurements for phase angles greater than 1.22 radians can be made with precise time-base-referencing methods.

These methods have not been applied as yet to the cyclic technique at Lewis, but the possibilities appear promising.

Two other areas that require consideration for extending the method to lower tem peratures are (1) the long time constants encountered and (2) the precise radiantintensity control at low-intensity levels. The long time constants increase the time over which absolute control of the entire experimental system must be maintained. Any undesirable thermal distrubance to the system can result in excursions of the sample temperature, which can nullify the data and require a restart of the experiment.

The problems of precise intensity control at the low intensities necessary to achieve low temperatures require that the electrical noise be minimized and that the instrumentation characteristics be carefully considered and used properly.

\section{Lewis Research Center,}

National Aeronautics and Space Administration, Cleveland, Ohio, May 3, 1968, 124-09-18-04-22. 


\section{APPENDIX - SYMBOLS}

A temperature amplitude during cyclic period, $\mathrm{K}$

$c_{\mathrm{p}} \quad$ specific heat, $\mathrm{J} /(\mathrm{g})(\mathrm{K})$

Io initial steady radiant intensity, $\mathrm{J} /\left(\mathrm{cm}^{2}\right)(\mathrm{sec})$

$i_{0} \quad$ intensity amplitude at mean temperature during cyclic equilibrium, $\mathrm{J} /\left(\mathrm{cm}^{2}\right)(\mathrm{sec})$

$\mathrm{k} \quad$ intensity perturbation factor

q heat exchange between sample and surroundings, $\mathrm{J} /\left(\mathrm{cm}^{2}\right)(\mathrm{sec})$

t time, sec

$\mathrm{T}$ temperature, $\mathrm{K}$

$\dot{\mathrm{T}}$ time rate of temperature change, $\mathrm{K} / \mathrm{sec}$

$\mathrm{T}_{\mathrm{m}} \quad$ mean temperature of sample, $\mathrm{K}$

$\alpha \quad$ absorptance

$\delta(\mathrm{u}) / \mathrm{u} \quad$ relative error term

$\epsilon \quad$ emittance

$\rho \quad$ material density, $\mathrm{g} / \mathrm{cm}^{2}$

$\theta^{\prime} \quad$ time constant of material, sec

$\sigma \quad$ Stefan-Boltzmann constant, $J /\left(\mathrm{cm}^{2}\right)($ sec $)\left(K^{4}\right)$

$\tau \quad$ material thickness, $\mathrm{cm}$

$\varphi \quad$ phase angle between sample temperature and cyclic incident radiation, rad

$\omega \quad$ cyclic frequency, $\mathrm{rad} / \mathrm{sec}$ 


\section{REFERENCES}

1. Jack, John R.: Technique for Measuring Absorptance and Emittance by Using Cyclic Incident Radiation. AIAA J., vol. 5, no. 9, Sept. 1967, pp. 1603-1606.

2. Mark, Herman: Operation of a Solar Space Environment Simulator. Proceedings of the Institute of Environmental Sciences, Washington, D.C., Apr. 5-7, 1961, pp. 521-531.

3. Terman, Frederick E.; and Pettit, Joseph M.: Electronic Measurements. Second ed., McGraw-Hill Book Co., Inc., 1952.

4. Gubareff, G. G.; Jansen, J. E. ; and Torborg, R. H. : Thermal Radiation Properties a Review of the Literature. Second ed., Honeywell Research Center, Minneapolis, Minn., 1960.

5. Touloukian, Y. S., ed.: Thermophysical Properties of High Temperature Solid Materials. Macmillan Co., 1967. 
The aromintid and spate activites of the United sintes shall be condtucted so as to contibite. to the expansion of bumm knoul. elge of phonomeno in the amaphere and spare. The Administration shall pronde for the witest pricticable and thpropriate dirsemination

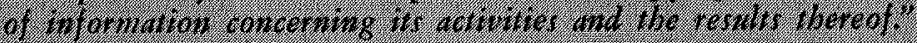

\section{NASA SCIENTIFIC AND TECHNICAL PUBLICATIONS}

TECHNICAL REPORTS: Scientefe and rechnical information considered important, complete, and a lasting contribition to existing knowiledge:

TICLNICAT NOTES Information less broad in scope but nevertheless of imparance is a contriburion to existing kiowledge

TEETINICAI MENOR.ANDUMS

Information teceiving limiced distribution beruse of preliminary thea, secutity entssifici. tha the cher restons.

CONTR ICTOR REPOR1S: Scientic and cethrical infommition generated under N NASA contract or grmi thit consiktered an important. contribution to existute knivetedge.
TECHNICAI TRANSI ATIONS: Information published in a foreign language constuered to nerit NASA distribution in tenglish.

SPECTAL PUBLICATIONS: Information derived from of of talue ta NASA activities. Pablicutions ind hude conference proceetings. monographs, dara compilations. handbooks. somrcehooks, and special bibliographies.

\section{TECKNOLOGY UTIZAMON}

PUbIICATIONS: Information on technology insed by NASA that may be of particulik

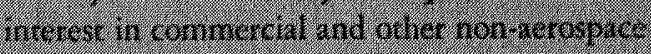
applications. Publications include Tedi Brtets. Technologr Utilizition Reports and Notes. ind Technologry Survicys.

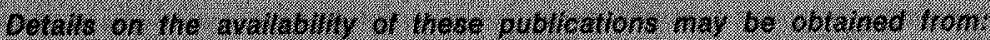

SCIETIEC AND TECINICAL INTORMATION DUSION

NATIONAL AERONAUTICS AND SPACE ADMINISTRATION Waltington, DC: 20546 